Mots. Les langages du politique

Discours de violence au nom de la foi

\title{
"Le mal existe ». Religion et néoconservatisme dans le discours de George W. Bush
}

André Corten

\section{CpenEdition}

Journals

Édition électronique

URL : https://journals.openedition.org/mots/1282

DOI : $10.4000 /$ mots. 1282

ISSN : 1960-6001

Éditeur

ENS Éditions

Édition imprimée

Date de publication : 1 novembre 2005

Pagination : $9-20$

ISBN : 2-84788-084-4

ISSN : 0243-6450

Référence électronique

André Corten, « « Le mal existe ». Religion et néoconservatisme dans le discours de George W. Bush », Mots. Les langages du politique [En ligne], 79 | 2005, mis en ligne le 28 mai 2008, consulté le 23 avril 2022. URL : http://journals.openedition.org/mots/1282 ; DOI : https://doi.org/10.4000/mots.1282 


\section{"Le mal existe.» Religion et néoconservatisme dans le discours de George W. Bush ${ }^{1}$}

Questionné au cours d'un débat sur son philosophe ou penseur préféré, George W. Bush déclara : "Le Christ»; et comme on lui demandait pourquoi, il répondit: "Parce qu'il a changé mon cœur.» Le président américain porte sa foi en bandoulière, mais le sénateur John Kerry n'est pas demeuré en reste lors de la dernière campagne électorale: «Je pense que tout ce que vous faites dans la vie publique doit être guidé par votre foi », a-t-il déclaré dans un débat où les mots Dieu, foi, religion, prier ont été prononcés quarante-deux fois.

Si Talcott Parsons, reprenant d'une certaine façon Tocqueville ${ }^{2}$, avait, il y a quarante ans, pu parler d'exception religieuse américaine (la religion étant vue comme garante, en Amérique, de la stabilité de la démocratie) ${ }^{3}$, aujourd'hui c'est plutôt d'exception européenne que l'on parle, en renvoyant à la sécularisation de ces sociétés ${ }^{4}$. Cela signifie-t-il que c'est la religion qui détermine désormais les enjeux politiques d'une bonne partie du monde, y compris des ÉtatsUnis? La manière dont les discours du président américain ont été répercutés dans les médias a contribué à cette lecture.

Dans la présente analyse, on montrera que les discours du président George W. Bush reposent plus sur une argumentation marquée par des notions appartenant à ce qu’on appelle la philosophie néoconservatrice (notamment

1. Cette étude, menée dans le cadre d'une réflexion sur «Sacré immanent et politique » par le Groupe de recherche sur les imaginaires politiques en Amérique latine - GRIPAL -, fait partie de travaux subventionnés par le Conseil de recherche en sciences humaines du Canada. Je remercie vivement Ginette Bergeron, qui a rassemblé les textes du corpus et les documents contextuels sur lesquels repose cette étude.

2. «Pour moi, je doute que l'homme puisse jamais supporter à la fois une complète indépendance religieuse et une entière liberté politique ; et je suis porté à penser que, s’il a une foi, il faut qu'il serve, et, s'il est libre, qu'il croie. » Tocqueville (de) A., 1981 [1840], De la démocratie en Amérique, t. 2, Paris, GF-Flammarion, p. 31.

3. F. Randaxhe, 2003, "De “l'exception religieuse” états-unienne. Retour sur un débat », Archives des sciences sociales des religions, $\mathrm{n}^{\circ} 122$, avril-juin, p. 7-25.

4. La sécularisation des sociétés européennes est une idée toute faite. On est plutôt en présence d'institutions ecclésiales quasi monopolistiques (Église catholique, Église anglicane...) perdant le contrôle de croyances religieuses devenues plus diffuses. Voir G. Davie, 2002, Europe: the Exceptional Case. Parameters of Faith in the Modern World, Londres, Darton, Longman and Todd Ltd.

Université du Québec à Montréal, GRIPAL, dept.sc.politiques@uqam.ca 
celle de Léo Strauss 5 ) que sur des notions religieuses à proprement parler. Lorsque ces dernières sont présentes, elles répondent à la nécessité de traduire en termes simples ("exotériques», dirait Strauss) les objectifs "ésotériques» de la philosophie politique. Néanmoins, les discours de George W. Bush ne fonctionnent pas comme pourraient l'attendre les disciples de Strauss. Le fondamentalisme n'étant pas vraiment, dans la société américaine, d'ordre religieux, mais d'ordre nationaliste, les quelques références au religieux ne produisent pas l'effet «exotérique » attendu. On verra au passage que George $W$. Bush ne transgresse pas réellement le partage du religieux et du politique tel qu'il est défini dans la tradition des États-Unis, mais s'appuie sur une religion civile, voire nationaliste, américaine. Les Églises contribuent à sacraliser l'expérience américaine, y compris en transférant la notion d'élection des saints à la nation, et déplacent ainsi la transcendance vers l'ici-bas ${ }^{6}$.

\section{Les discours de George W. Bush et le courant évangélique américain}

Tel qu'il est repris dans la presse, le discours de George W. Bush semble s'adresser au courant évangélique. Depuis un quart de siècle, ce courant a connu une expansion exceptionnelle dans de nombreuses régions du monde, y compris en Chine. Dans sa composante pentecôtiste, il a souvent été l'expression de l'émotion des pauvres7. Lorsqu'il s'agit des États-Unis, on l'étiquette généralement comme conservateur. Depuis Ronald Reagan, mais plus encore avec George W. Bush, le courant fondamentaliste, qui atteindrait près de $40 \%$ de la population (en fait, il s'agit du pourcentage de pratiquants toutes confessions confondues), semble colorer la politique américaine. Désormais, aux États-Unis, les incroyants se plaignent d'être persécutés par la majorité morale. Ils ont trouvé des porte-paroles qui parlent de «croisade» en s'appuyant sur l'emploi de ce terme par George W. Bush au lendemain du 11 septembre ${ }^{8}$.

À la suite de Robert N. Bellah', plusieurs spécialistes américains ont centré leur analyse sur la religion nationaliste américaine. Isabelle Richet, dans son

5. Philosophe politique d'origine juive allemande (1899-1973), professeur à l'université de Chicago. Voir notamment Droit naturel et histoire, 1988 [1953], Paris, Flammarion ; Nihilisme et politique, 2001 [1941, 1962], Paris, Rivages poche.

6. I. Richet, 2001, La religion aux États-Unis, Paris, PUF, Que sais-je, n- 3619, p. 107.

7. A. Corten, 1995, Le pentecôtisme au Brésil: émotion du pauvre et romantisme théologique, Paris, Karthala; P. Freston, 2001, Evangelicals and Politics in Asia, Africa and Latin America, Cambridge, Cambrige University Press.

8. B. Victor, 2004, La dernière croisade : les fous de Dieu version américaine, traduit de l'américain (The last crusade, Constable and Robinson) par F. Israël, Paris, Plon.

9. Dans son célèbre article de 1967, «Civil Religion in America », R. N. Bellah empruntait ses arguments à Durkheim et Rousseau. 
"Que sais-je », fait écho à ces travaux. Les deux récents livres de Sébastien Fath ${ }^{10}$ ont proposé une évaluation pondérée de l'influence des évangéliques aux États-Unis. En évoquant les figures de Martin Luther King ou de Jimmy Carter, Fath rappelle que cette influence n'est pas nouvelle et qu'elle n'a pas toujours été jugée sectaire.

Dans l'histoire américaine, la propagation du courant évangélique a des racines diverses et anciennes. Son influence en politique est plus récente, mais pas à proprement parler nouvelle : elle date de la seconde moitié des années soixante-dix. Après Jimmy Carter, un évangélique fervent mais jugé trop libéral, la majorité morale porte Ronald Reagan à la présidence en 1981. Lui succède son vice-président, George H. Bush. Celui-ci, en butte lors des primaires de 1988 à la candidature de Pat Robertson (fondateur de la Coalition chrétienne et célèbre télévangéliste de confession baptiste) ${ }^{11}$, fait appel à son fils, George W. Bush, pour organiser les contacts avec les milieux évangéliques. La conversion de ce dernier (1985) est connue dans son milieu (milieu patricien où on est plutôt épiscopalien), mais elle n'a été ni aussi subite, ni aussi radicale qu'on le prétendra. En outre, elle se fait dans le cadre d'une Église qui n'est pas particulièrement conservatrice, l’Église méthodiste unie ${ }^{12}$, Église qui par la suite marquera sa désapprobation face à la guerre en Irak.

Dans la présente analyse, on montrera que les discours de George W. Bush arrivent à articuler le religieux et le politique dans une formule choc: «Le mal existe ». Or, cette assertion, qui est le ressort fondamental du discours du président Bush, n'a pas principalement une base religieuse: elle repose sur la croyance dans le caractère universel des valeurs américaines (devenues synonymes de démocratie), et sur la conviction qu'il y aura toujours une résistance irréductible à celles-ci : nous faisons face, dit George W. Bush, à des ennemis qui haïssent nos valeurs et les espoirs de l'humanité. Une ontologie nouvelle émerge dès lors au plan politique. Elle est fondée sur la conception néoconservatrice du caractère inconciliable de la raison et de la Révélation, et s'enracine dans le mysticisme juif. Ce caractère inconciliable (Athènes et Jérusalem) est aussi pour Strauss la racine de l'institution du politique.

L'expression Empire du Mal faisait déjà partie du discours de Ronald Reagan, et y avait été introduite par un personnel politique qui s'est partiellement retrouvé dans l'entourage du président actuel (on attribue cette expression à

10. S. Fath, 2004, Dieu bénisse l'Amérique : la religion de la Maison-Blanche, Paris, Le Seuil ; 2004, Militants de la Bible aux États-Unis : évangéliques et fondamentalistes du Sud, Paris, Autrement.

11. Sur le télévangélisme (prédication par la télévision, qui semble avoir connu son apogée entre 1980 et 1985), voir notamment J. Gutwirth, 1998, L'Église électronique. La saga des télévangélistes, Paris, Bayard.

12. Le méthodisme, fondé par Wesley à la fin du $18^{\mathrm{e}}$ siècle, se répand d'abord en milieu ouvrier anglais et arrive rapidement aux États-Unis. Prenant ses distances vis-à-vis de la doctrine de la prédestination prêchée par Calvin, le méthodisme accorde une attention à l'autonomie humaine. 
Richard Perle, considéré comme un straussien). Néanmoins, la formule avait perdu de son impact pour deux raisons : l'écroulement de l'Empire soviétique et, en politique intérieure, l'affaiblissement des républicains. Par ailleurs, la conception dispensationaliste ${ }^{13}$ que connotait la notion d'Empire du Mal, teintée de prémillénarisme et donc d'une vision de fin du monde, était devenue peu compatible avec ce que certains appellent la « fin de l'Histoire », traduite dans la prétendue victoire du libéralisme économique et de la démocratie. L'expression Empire du Mal faisait partie d'un discours littéral de diabolisation : l'Empire du Mal était celui de Satan et la bataille ultime celle d'Armageddon ${ }^{14}$.

La notion d'axe du mal (à noter que mal n'a plus de majuscule) n'est pas millénariste. Elle s'articule à une perspective de philosophie politique qui, à partir d'une conception du meilleur régime possible, prétend distinguer l'existence du mal et définir par là le bien pour l'humanité. Les États-Unis se donnent ainsi le droit d'imposer la démocratie. À ce sujet, une polémique a traversé l'Atlantique en 2003 à partir des écrits d'un personnage contesté, Lyndon LaRouche ${ }^{15}$. Partant du New Yorker, elle a envahi les principaux magazines européens. L'affirmation selon laquelle l'ultraconservatisme de la politique américaine est directement inspiré de la pensée de Léo Strauss a suscité une levée de boucliers. Les images d'Épinal se sont bousculées. Oui ou non, la pensée du maitre a-t-elle, notamment à travers son disciple Allan Bloom, essaimé dans les hautes sphères de l'administration américaine? On désigne le numéro deux du Pentagone, Paul Wolfowitz, nommé depuis lors directeur de la Banque mondiale. La polémique éclate en milieu francophone au moment où le roman de Saul Bellow, Ravelstein ${ }^{16}$ (qui vient d'être traduit en français), popularise l'image de philosophes invoquant Platon ou Aristote et téléphonant du même souffle aux artisans de la politique américaine.

13. Dispensation, en termes bibliques, signifie voie (ou période) de la Révélation. Au $19^{\mathrm{e}}$ siècle se répand une doctrine sur la base d'une interprétation particulière des sept dispensations déjà élaborées par le moine cistercien Joaquim de Flore au $12^{\mathrm{e}}$ siècle. Cette interprétation dispensationaliste conditionne l'attitude actuelle de beaucoup de chrétiens américains vis-à-vis d'Israël. En effet, les diverses tendances d'interprétation du dispensationalisme s'accordent sur la nécessité du retour des juifs en Israël si l'on veut voir advenir le second Retour du Christ. Selon certaines interprétations, le temple de Jérusalem sera reconstruit, puisque des prophéties annoncent sa destruction par l'Antéchrist. Tandis que l'Église sera «enlevée» dans le ciel, la terre connaitra sept années de tribulations durant lesquelles se livrera une bataille décisive entre le Bien et le Mal. Le rapport entre l’Église et les juifs est au cœur des controverses théologiques au sujet du dispensationalisme.

14. M. Ben Barka, 1998, Les nouveaux rédempteurs. Le fondamentalisme protestant aux États-Unis, Genève, Labor et Fidès, p. 97-106.

15. Candidat marginal aux primaires démocrates américaines de 1999.

16. Publié en 2000, traduit de l'américain par Rémy Lambrechts chez Gallimard en 2002. Le livre raconte entre autres la genèse et le succès incroyable du livre d'Allan Bloom (1930-1992), 1988, The Closing of the American Mind, Simon \& Schuster (traduit en français par P. Alexandre, 1987, L'Âme désarmée, Paris, Julliard), dans lequel l'auteur s'adonne à une critique féroce du relativisme dans les milieux académiques américains. 
Revenons au religieux dans le discours de George W. Bush. On répète à l'envi que le président invoque systématiquement Dieu dans ses discours, et qu'aucun président américain n’a autant utilisé la théologie. On fait aussi constamment référence au terme de croisade prononcé lors de sa conférence de presse du 16 septembre $2001^{17}$. Enfin, à l'occasion des élections présidentielles de novembre 2004, on a fait état du soutien massif des évangéliques à sa candidature. En fait, selon l'interprétation straussienne, on a souvent eu recours au langage religieux pour faire passer des idées qui, sinon, seraient incompréhensibles au grand public ${ }^{18}$.

L'analyse de quelques grands discours du président George W. Bush (les discours devant l'ONU et sur l'état de l'Union, auxquels a été ajouté le discours devant le Congrès du 20 septembre 2001) conduit à une lecture nuancée de l'importance du religieux dans la politique américaine et permet de mesurer comment, derrière le fondamentalisme, s'installe une argumentation néoconservatrice. Dans cette analyse du discours inscrite dans la tradition de l'école française ${ }^{19}$, on s'intéressera d'abord à la fondation de la deixis du 11 septembre, ensuite à l'environnement argumentatif des syntagmes à caractère religieux et enfin, à la mise en discours des valeurs américaines.

\section{La fondation d'une deixis}

La force du discours de George W. Bush réside dans sa capacité à établir une coupure, aujourd'hui largement acceptée dans le monde, entre l'avant et l'après 11 septembre 2001. Cette date devient la référence ; elle est continuellement remise en scène et réactive la légitimité du discours. Elle fonde une deixis.

Des marques déictiques (surtout temporelles) scandent le discours du 21 septembre 2001. L'incipit déjà exprime en implicite une rupture: «En temps normal », dit George W. Bush pour affirmer qu'on est en temps anormal. S'oppose à cette expression la formule ce soir répétée de nombreuses fois. La partie finale du discours contient cette phrase:

J'espère que dans les mois et les années à venir, nous renouerons avec une vie presque normale. [...] Chacun d'entre nous se souviendra de cette journée, et à qui

17. «Le temps est maintenant venu de gagner de manière décisive la première guerre du $21^{\mathrm{e}}$ siècle. Cette croisade, cette guerre contre le terrorisme demandera du temps. »

18. Notons que Strauss se distingue de Spinoza, selon qui le religieux et le prophétique ne sont utiles que pour ceux qui n'ont pas accès à la raison. Strauss a une position moins rationaliste. Pour lui, le maniement de l'exotérique et de l'ésotérique est un art: L. Strauss, 1989 [1953], La persécution ou l'art d'écrire, Paris, Pocket. Voir C. Lefort, 1992, Écrire. À l'épreuve du politique, Paris, Calmann-Lévy, p. 261-301.

19. Telle qu'elle est systématisée, notamment, par D. Maingueneau, 1997 (L'analyse du discours, Paris, Hachette) et mise en perspective par J. Guilhaumou, D. Maldidier et R. Robin, 1994, Discours et archive, Liège, Mardaga. 
c'est arrivé. Nous nous souviendrons du moment où nous avons appris la nouvelle où nous étions et ce que nous faisions. Certains garderont la mémoire d'un visage et d'une voix à jamais disparus.

On a une normalité d'avant le 11 septembre. On espère qu'il y aura une normalité d'après le 11 septembre. Entre les deux prend place un discours chargé d'une grande émotivité. Il en est de même dans le discours sur l'état de l'Union prononcé trois mois plus tard (30 janvier 2002) ${ }^{20}$, où l'on constate à nouveau l'importance du mot normal:

La dernière fois que j'ai pris la parole ici, j'ai exprimé l'espoir que la vie reviendrait à la normale. À certains égards, elle l'est redevenue. À d'autres, elle ne le redeviendra jamais. Ceux d'entre nous qui ont été touchés par ces dures épreuves en sont sortis changés. Nous avons appris des vérités dont nous ne douterons jamais, à savoir que le mal existe et qu'il faut s'y opposer. [...] En un instant, nous nous sommes rendu compte que cette décennie serait décisive dans l'histoire de la liberté et que nous étions appelés à jouer un rôle exceptionnel dans le cours des évènements de l'humanité.

Tous les discours aux Nations unies font référence au 11 septembre, souvent en incipit. Ainsi dans le discours du 10 novembre 2001: "Nous voici réunis dans une salle qui se consacre à la paix, dans une ville qui porte les cicatrices de la violence... ». Dans le discours du 21 septembre 2004, la référence intervient dans le deuxième paragraphe : «Au cours des trois dernières années, je me suis adressé à cette Assemblée en des temps tragiques pour mon pays... ».

Dans les discours sur l'état de l'Union, la référence est souvent indirecte: "Vous et moi servons notre pays à une époque lourde de conséquences» (30 janvier 2002). Mais dans celui du 2 février 2005, la deixis fondatrice est encore inscrite dans les lignes qui introduisent et qui concluent la partie consacrée à la politique internationale: «Au cours des trois années et demie qui se sont écoulées depuis le 11 septembre 2001... "; «Au cours des quatre dernières années, les Américains ont été témoins d'évènements de grande ampleur ».

\section{Les prédicats des énoncés à caractère religieux}

Les expressions comportant un vocabulaire religieux sont inégalement réparties dans les discours. C'est juste après le 11 septembre que le nombre d'occurrences est le plus élevé. Les références à Dieu et à la religion s’inscrivent dans deux contextes: d'une part, il est fait référence à l'islam, voire à toutes les

20. Traduction officielle du Département d’État disponible en novembre 2004 sur Internet: usinfo.state.gov/français. En version originale: usinfo.state.gov. 
religions, et de l'autre, au dieu des chrétiens, bien qu'il ne soit pas nommé. On relève donc deux paradigmes relatifs à l'invocation de Dieu. Dans le premier, où il s'agit de l'islam et des autres religions (l'expression grandes religions est répétée systématiquement), il s'agit d'affirmer que Dieu rejette tant le meurtre d'innocents que le suicide, et qu'il est inapproprié de mettre, avec le mot martyrs, victimes et bourreaux sur le même plan. Aucune appréciation négative n'est portée sur le dieu de l'autre. Bien mieux, le discours peut s'engager dans une démarche inclusive. La référence au même dieu donne alors plus de force à la condamnation:

Ils osent demander la bénédiction de Dieu alors qu'ils s'apprêtent à tuer des hommes, des femmes et des enfants innocents. Mais le Dieu d'Isaac et d'Ismaël ne répondrait jamais à une telle prière. (Nations unies, 10 novembre 2001).

Le second paradigme décrit le dieu de la religion nationaliste américaine. On remarque les connotations euphoriques colportées par les prédicats : Dieu est engagé, proche, il donne, il est une source, il aime, il bénit. Sur le plan argumentatif, rien de périlleux; il s'agit de renforcer les valeurs américaines :

La liberté et la peur, la justice et la cruauté se sont toujours fait la guerre, et nous savons que, dans cette lutte, Dieu n'est pas neutre. (Congrès, 20 septembre 2001)

Les Américains forment un peuple libre, qui sait que la liberté est le droit inné de chaque être humain et l'avenir de toute nation. La liberté que nous chérissons n'est pas le don de l'Amérique au monde, c'est le don de Dieu à l'humanité. (État de l'Union, 29 janvier 2002)

Ces énoncés ont pour fonction d'associer Dieu à l'Amérique. Ils contribuent au renforcement d'une religion nationaliste américaine, qui tend à être présentée comme universelle :

Nous sommes aussi certains que l'histoire a un auteur qui remplit le temps et l'éternité avec ce but. Nous savons que le mal est réel, mais que le bien l'emportera. C'est là l'enseignement de nombreuses religions. (Nations unies, 10 novembre 2001)

Pourtant, il est erroné, et condescendant, de postuler que des cultures entières et de grandes religions sont incompatibles avec la liberté et avec l'autonomie. Je crois fermement que Dieu a planté en chaque âme le désir de vivre libre. Et même quand ce désir est étouffé par la tyrannie pendant des décennies, il ressurgit toujours. (État de l'Union, 20 janvier 2004)

La seule force assez puissante pour stopper la montée de la tyrannie et de la terreur et remplacer la haine par l'espoir est le désir de liberté des hommes. (État de l'Union, 2 février 2005)

L'invocation de Dieu affirme une loi naturelle: la liberté est dans la nature humaine, alors que la tyrannie est opposée à la volonté divine. L'opposition de départ, celle du bien et du mal, glisse ainsi vers une opposition liberté/mal, car la liberté appartient aux valeurs américaines. Celles-ci sont le bien. 


\section{Mise en discours des valeurs américaines}

Plus que les paroles de George W. Bush sur l'état de l'Union, celles qu'il prononce devant les Nations unies permettent de mettre en discours les valeurs américaines. Le processus repose sur la répétition de certains couples: bien/mal, civilisation/terrorisme, dignité de la vie/culture de la mort, changement dans la légalité/subversion, règles du droit/dérégulation, paix du monde/violence, sécurité/menace, liberté/oppression, confiance/destruction, justice/injustice, droit naturel/État totalitaire, espoir/peur, développement/misère, élections supervisées/chefs autodésignés, tolérance/sectarisme, respect des femmes/ brutalité envers les femmes, échanges commerciaux/isolement, écrire l'histoire/ n'avoir aucune place, droits inaliénables/meurtres délibérés d'innocents, gouvernement honnête/corruption, progrès/faim, vérité/mensonges, etc. Chaïm Perelman et Lucie Olbrechts-Tyteca ${ }^{21}$ ont montré comment l'alignement de couples notionnels, tout en donnant l'impression de ne rien apporter de neuf, permet des remaniements et des glissements - témoin, plus haut, le passage de la notion de bien à celle de liberté. De son côté, Umberto $\mathrm{ECO}^{22}$ a mis en évidence, à un autre propos, l'effet idéologique produit par la mise en œuvre d'invariants. Pour rendre compte de la bonne réception du discours de George W. Bush dans le public américain, on ne peut pas se référer en premier lieu à un manichéisme qui serait consubstantiel au fondamentalisme. Au contraire, l'opposition d'invariants permet de souder l'électorat républicain et de donner aux évangéliques une place active dans celui-ci.

La religion nationaliste américaine est la manière d'identifier les Américains par rapport au reste du monde. Aussi, les couples notionnels sont beaucoup plus nombreux dans les discours prononcés aux Nations unies que dans les discours sur l'état de l'Union. C'est aussi vis-à-vis de l'extérieur que la «vie bonne » (de la démocratie, forcément américaine) doit être opposée au mal comme facteur réel.

Dans les discours sur l'état de l'Union, la référence au religieux est peu fréquente. Mais lorsqu'elle a lieu, un lien explicite est établi entre le religieux et les valeurs fondamentales américaines ou les États-Unis :

Nous ne prétendons pas connaitre toutes les voies de la Providence, pourtant nous pouvons lui faire confiance, et placer tous nos espoirs en ce Dieu aimant qui est source de toute vie, et de toute l'histoire.

Puisse-t-Il nous guider aujourd'hui, et continuer de bénir les États-Unis d’Amérique. (État de l'Union, 29 janvier 2003)

21. C. Perelman et L. Olbrechts-Tyteca, 1958, La nouvelle rhétorique. Traité de l'argumentation, Paris, PUF, p. 569.

22. U. Eco, 1966, "James Bond: une combinatoire narrative», Communications, $\mathrm{n}^{\circ} 8$, L'analyse structurale du récit, p. 83-89. 
Notre génération a ses rêves [...]. La route de la Providence [...] aboutit à la liberté. (État de l’Union, 2 février 2005)

\section{Les limites d'une ontologie politique postmachiavélienne}

Avec Machiavel, le mal est considéré comme nécessaire mais, si on peut le prendre comme il faut, fécond ${ }^{23}$. La souffrance ou le bonheur dépendent de la Fortune, mais l'être humain peut dans une certaine mesure contrecarrer celle-ci. Pour Léo Strauss, Machiavel ${ }^{24}$, contrairement à Spinoza, a au moins le courage d'affronter la terreur originelle. Il y a chez lui une quasi-théologie puisque la Fortune (plus que la chance, la Fortune désigne la situation historique favorisant plus ou moins les projets du Prince) y remplace Dieu; mais Machiavel renverse complètement la théologie chrétienne en abandonnant son aspect téléologique qui donnait au Bien un statut premier. Le mal occupe dès lors une place nouvelle dans sa cosmologie et la nature est considérée comme une ennemie à mater. La colère anti-théologique machiavélienne, comme celle plus tard de Hobbes, résulte en partie de son observation de la «cruauté pieuse» des guerres de religions, et en particulier de l'expulsion des marranes d'Espagne. En prétendant que l'action politique n'est pas orientée vers un bien, Machiavel mine les prétentions de l’Église à intervenir dans celle-ci. Par contre, il perpétue comme d'autres une tradition enracinée, celle de l'écriture exotérique. On pense «Fortune », mais on parle de Providence.

Pour Strauss, la pensée politique moderne fait perdre au mal son enjeu tragique. C'est désormais le moindre mal qui devient le guide de l'action politique. Le mal n'a plus dès lors de contenu ontologique et se dilue dans le relativisme des valeurs. "Il y a eu le nihilisme destructeur et guerrier du nazi, et il y a le nihilisme mou du relativisme contemporain. ${ }^{25}$ George W. Bush se défend de tomber dans ce nihilisme mou. « Nos ennemis croyaient que l'Amérique était faible et matérialiste, que la peur et l'égoïsme nous feraient craquer» (État de l'Union, 29 janvier 2002). L'affirmation «le mal existe» est en effet à l'opposé de la notion de moindre mal. Pourtant, toute la construction discursive sur la deixis fondatrice conduit au résultat contraire et aboutit à produire l'effet de sens suivant : ce sont nos ennemis qui nous permettent de retrouver nos valeurs. En d'autres termes, ce ne sont pas les valeurs qui sont premières, ce sont les ennemis qui font se resserrer le peuple américain, et même l'humanité entière, sur des valeurs :

23. P. Manent, 1987, «Machiavel et la fécondité du mal », Histoire intellectuelle du libéralisme. Dix leçons, Paris, Calmann-Lévy, chap. 2.

24. Pensée sur Machiavel, p. 187, cité par D. Tanguay, 2003, Léo Strauss : une biographie intellectuelle, Paris, Grasset, p. 180.

25. L. Strauss, 2001, Nihilisme et politique, op. cit., p. 19. 
Comme je l'ai dit à mes compatriotes, la liberté et la peur sont en guerre. Nous faisons face à des ennemis qui haïssent non pas notre politique, mais notre existence, la tolérance de l'ouverture au monde extérieur et la culture créative qui nous caractérise. Toutefois, l'issue de ce conflit est certaine. On observe un courant dans l'histoire du monde, et ce courant va vers la liberté. Nos ennemis le rejettent, mais les rêves de l'humanité sont définis par la liberté, par le droit naturel de créer, de bâtir, de prier et de vivre dans la dignité. Lorsque les hommes et les femmes sont libérés de l'oppression et de l'isolement, ils ont un sentiment de plénitude et d'espoir et ils sont des millions à sortir de la pauvreté. Nous défendons les espoirs permanents de l'humanité, et on ne peut pas refuser ces espoirs. (Nations unies, 10 novembre 2001)

L'attaque lancée contre la liberté dans notre monde a réaffirmé notre confiance dans le pouvoir qu'a la liberté de changer le monde. (État de l'Union, 2 février 2005)

Ce disant, George W. Bush laisse transparaitre un propos plus schmittien que straussien. Strauss avait pourtant, dans une célèbre polémique ${ }^{26}$, critiqué le caractère formel ou vide (en termes de valeurs) de l'opposition ami/ennemi que Carl Schmitt considère comme le critère du politique ${ }^{27}$. Toute l'insistance donnée à la deixis fondatrice du 11 septembre affaiblit en fait l'énoncé ontologique selon lequel « le mal existe».

Le discours affirme donc que le mal existe, mais son fonctionnement évide celui-ci de son caractère ontologique. Ce vide est alors rempli par deux types de signes : le mot liberté, ainsi qu'une série de termes relatifs à la religion. Liberté est généralement employé absolument, sans génitif ni adjectif. Ce mot qui a permis, durant la guerre froide, de concevoir la division du monde, libre d'un côté et opprimé de l'autre, est central dans la Déclaration d'indépendance de 1776 :

Nous tenons pour évidentes pour elles-mêmes les vérités suivantes: tous les hommes sont créés égaux; ils sont doués par le Créateur de certains droits inaliénables; parmi ces droits se trouvent la vie, la liberté et la recherche du bonheur.

Dans les discours de George W. Bush, si liberté intervient constamment, droit à la vie (sauf dans le sens de «culture du respect de la vie», État de l'Union, 2 février 2005) n'apparait que rarement, et recherche du bonheur pas du tout. En sortant la liberté de l'ensemble des autres droits, on fait de ce mot l'équivalent du terme plus général, et souvent répété aussi, de droits de l'homme ${ }^{28}$. Le vide ontologique est davantage rempli par des termes comme liberté que par la référence au religieux. Le fait d'avoir pris cette référence comme sujet de

26. H. Meier, 1990, Carl Schmitt, Léo Strauss et la notion de politique: un dialogue entre absents, Paris, Julliard. Selon Strauss, le caractère en fin de compte formel de l'opposition ami/ennemi amène Schmitt à fonder paradoxalement sa critique du libéralisme sur Hobbes, qui est précisément le fondateur du libéralisme. Pour Strauss, le mal n'est pas de l'ordre de la nature (comme chez Hobbes, position que Schmitt assume sans l'expliciter), mais justement de la violation des lois naturelles. II n'a pas un caractère anthropologique, mais ontologique.

27. C. Schmitt,1992, La notion du politique, Paris, Flammarion (Champs).

28. M. Gauchet, 2002, La démocratie contre elle-même, Paris, Gallimard, p. 1-26. 
notre article ne doit donc pas prêter à confusion : malgré le rôle de celui qui est surnommé le «scribe » par son patron (Michael Gerson, son speech-writer, un évangélique convaincu), la référence au religieux est faible dans les discours officiels. Le président Bush, respectant son rôle constitutionnel, ne désigne Dieu que comme le Créateur ou la Providence. La seule fois où il s'est écarté de cette attitude dans les discours qui font l'objet de cette analyse, c'est lorsqu'il a réuni dans un même propos le dieu d'Israël et d'Ismaël.

Ce monde est formé en partie de militants de la Bible. C'est tout l'intérêt du livre de S. Fath ${ }^{29}$ de montrer comment l'imaginaire, si fort au $19^{\mathrm{e}}$ siècle, s'est figé à partir des années soixante-dix. L'imaginaire du religieux n'est plus un renvoi continuel de significations comme ce fut le cas dans la lutte contre l'esclavage ou la campagne des droits civiques. II n'est pas animé par une mystique de l'irréductible. Il s'est plutôt sécularisé et coagulé sur les paramètres d'une Amérique profonde. Il est sans doute anti-gay et anti-avortement, mais moins pour des raisons religieuses que par une sorte de chauvinisme localiste. Le revivalisme est du passé, on conserve la religion comme mode de protestation contre l'internationalisation de la politique, en particulier états-unienne, car les dirigeants les plus isolationnistes se métamorphosent une fois arrivés au pouvoir. L'énoncé le mal existe produit un renvoi de significations à des affaires relevant de faits divers. Il connote une volonté essentiellement réactive. Aussi énergique que semble cet énoncé, sa force illocutoire est donc modeste. Bien que straussien en apparence, il est paresseux lorsqu'il s'insère dans le discours de sens commun, auquel Strauss préconisait pourtant de revenir pour fonder le politique. En fin de compte, l'ésotérique ne donne pas de ressort à l'exotérique. Quand le discours se sera complètement déroulé, on se rendra compte que le roi est nu. L'approche straussienne, qui semblait dotée d'une puissance légitimante extraordinaire, s'avère fonctionner en bout de course comme une réaction formelle à un ennemi tout aussi vide.

George W. Bush lit un passage de la Bible tous les jours. Il prie avant chaque réunion importante. Il professe des actions de grâce en conférence de presse. On rapporte qu'il ne prononce pas un discours sans référence biblique... et ne manque pas de raconter son histoire personnelle avec Dieu. Le 16 septembre 2001, le président américain a appelé «tous ceux qui portent l'uniforme » à se tenir prêts pour une longue «croisade contre le terrorisme ».

Or, c'est là le seul emploi de ce mot. Notre analyse, qui porte sur des contextes où les notions religieuses interviennent, permet aussi de constater que celles-ci restent peu nombreuses dans les discours de George W. Bush. La référence à ces notions relève d'ailleurs d'une tradition : les textes fondateurs

29. S. Fath, 2004, Militants de la Bible..., op. cit., chap. 9, p. 198. 
en sont parsemés; les prédécesseurs en ont usé (on cite en particulier à cet égard le président Thomas Woodrow Wilson, 1912-1920) ${ }^{30}$. Dans les discours de George W. Bush aux Nations unies et au Congrès (2001-2005), différents mécanismes discursifs font glisser les notions concernant le divin vers ce que l'on appelle la religion nationaliste américaine.

30. S. Fath, 2004, Dieu bénisse..., op. cit., p. 184. Toujours selon Fath (p. 103), «l'influence dominante du protestantisme évangélique sur la “couleur" de la religion civile américaine actuelle... ne date pas de George W. Bush puisqu'elle est construite dès la présidence du démocrate Lyndon Johnson (1963-1968), particulièrement proche du prédicateur Billy Graham. » À ma connaissance, il n'existe pas d'étude comparant systématiquement l'usage de notions religieuses dans les discours des présidents américains. 